\title{
Capture in Nonlinear Differential Games of Pursuit
}

\author{
Dr.Musa T.Y.Kadzai ${ }^{1}$, Prof.E.N.Chukwu \\ ${ }^{I}$ Department of Mathematics, Modibbo Adama University of Technology, Yola, Nigeria \\ ${ }^{2}$ Formerly: Professor of Mathematics, University of Jos, Jos, Plateau State, Nigeria \\ Currently: Professor Emeritus of Mathematics, North Carolina State University, USA
}

\begin{abstract}
We look at a brief historical survey of controllability problems of time optimal control theory. We next look at nonlinear variation of parameters to investigate controllability of basically nonlinear systems whereby we establish nonlinear differential games of pursuit results.
\end{abstract}

Keywords: Optimal Control, Controllability, Games of Pursuit

\section{Introduction}

Modern Optimal Control Theory began in 1953 when Bushaw in a Ph.D. Thesis at Princeton, gave a mathematical solution to a simple optimal control problem. Hermes and Lasalle [1] showed that Bushaw considered the general second-order linear differential equation with constant coefficients

$$
\ddot{x}_{1}+2 b_{0} \dot{x}_{1}+c_{0} x_{1}=u(t)
$$

which is equivalent to

$$
\dot{x}=A x+B u
$$

where

$$
A=\left[\begin{array}{cc}
0 & 1 \\
-c_{0} & -2 b_{0}
\end{array}\right], B=\left[\begin{array}{l}
0 \\
1
\end{array}\right]
$$

and $|u| \leq 1$. The problem is to determine a control $u^{*}$ with $\left|u^{*}\right| \leq 1$ in such a way that the solution $x\left(t ; u^{*}\right)$ of (1) reaches the origin in $E^{2}, 2$-dimensional Euclidean space, in minimum time $t \geq 0$. Such a control will be termed,

Lasalle [2] considered a more general situation (2) in which A is an nxn matrix, B an nxm matrix and $u$ an m-vector which is constrained to lie in a unit cube $C^{m}$ in $E^{m}$ where $E^{m}$ is the m-dimensional Euclidean space. Here $C^{m}=\left\{u \in E^{m}:\left|u_{j}\right| \leq 1, j=1, \cdots, m\right\}$. The admissible controls are those whose components $u_{j}, j=1, \cdots, m$ are measurable on finite intervals with $-1 \leq u_{j}(t) \leq 1, t \geq 0$.

In tackling the time optimal control problem (2) Lasalle showed that if the system (2) has a solution which can be driven to zero with an admissible control in finite time (i.e. if the system (2) is null controllable) then optimal controls exist for (2) and can sharply be determined and described. The problem of null controllability was therefore of fundamental importance. Lasalle's solution was one of the earlier triumphs of Optimal Control Theory. He proved that if the free system

$$
\dot{x}=A x
$$

Is stable (i.e. all the eigenvalues of $A$ have non positive real part) and if the system (2) is proper i.e.

$\operatorname{Rank}\left[\mathrm{B}, \cdots, A^{n-1} B\right]=\mathrm{n}$

then the system (2) is null controllable. If we apply this theory to Bushaw's problem we note that since

we have

$$
b_{0}>0, c_{0}>0
$$

$$
\ddot{x}_{1}+2 b_{0} \dot{x_{1}}+c_{0} x_{1}=0
$$

is stable. Furthermore we have

$$
\operatorname{rank}[\mathrm{B}, \mathrm{AB}]=2
$$

where

$$
B=\left[\begin{array}{l}
0 \\
1
\end{array}\right] \text { and } A=\left[\begin{array}{cc}
0 & 1 \\
-c_{0} & 2 b_{0}
\end{array}\right]
$$

we have therefore that (2) is null controllable, optimal controls exist for (2) and can fully be described.

Lasalle's problem of null controllability has since been extended to nonlinear ordinary differential systems

$$
\dot{x}(t)=f(t, x(t), u(t)) .
$$

Lee and Markus [3] studied the nonlinear autonomous control problems

$$
\dot{x}=f(x, u) \text { in } C^{1} \text { in } E^{n+m}
$$

with $u$ admissible. They demonstrated that if

$$
\begin{aligned}
& f(0,0)=0 \\
& \operatorname{rank}\left[B, A B, \cdots, A^{n-1} B\right]=n
\end{aligned}
$$


where

$A=\frac{d f}{d x}(0,0)$ and $B=\frac{d f}{d u}(0,0)$

then there is an open region about the origin for which (11) is null controllable. By applying some asymptotic criterion they deduced global null controllability of the system (11). They applied the theory developed to the system

$$
\ddot{x}+f(x, \dot{x}) \dot{x}+g(x)=u
$$

or the phase plane system

$$
\begin{aligned}
& \dot{x}=y \\
& \dot{y}=-g(x)-f(x, y) y+u .
\end{aligned}
$$

The problem is the regulation of the nonlinear oscillator (15). They showed that if,

$$
\begin{aligned}
& f(x, y)>0 \\
& x g(x)>0 \text { for } x \neq 0
\end{aligned}
$$

and

$$
\begin{aligned}
& \lim _{|x| \rightarrow \infty} G(x)=\infty \\
& G(x)=\int_{0}^{x} g(s) d s,
\end{aligned}
$$

the linear approximation to the nonlinear control process at the origin is

where

$$
\dot{X}=\left[\begin{array}{cc}
0 & 1 \\
\frac{d g}{d x}(0) & -f(0,0)
\end{array}\right] X+\left[\begin{array}{l}
0 \\
1
\end{array}\right] u
$$

$$
X=\left[\begin{array}{l}
x \\
y
\end{array}\right]
$$

satisfies the controllability condition; thus, the nonlinear oscillator is controllable to the origin from each initial state in finite time by a controller $\mathrm{u}(\mathrm{t})$ satisfying any previously assigned bound $|u(t)|<\epsilon$.

The extension of the results of [3] to the nonlinear system (10) was attained in Chukwu [4]. Chukwu considered (10) where $f: R \times R^{n} \times R^{m} \rightarrow R^{n}$ is such that $\mathrm{f}(\mathrm{t}, \mathrm{x}, \mathrm{u})$ and $\frac{\partial f}{\partial x}$ are continuous in (t, $\left.\mathrm{x}, \mathrm{u}\right), u \in \Omega, \Omega$ the set of all bounded measurable controls with values in $\Omega \in R^{m}$, m-dimensional real space. He showed that if for some admissible control $u^{*}(t) \in \Omega$,

(i) $\quad \lambda_{k}\left(x, u^{*}, t\right), k=1,2, \cdots, n$ of the matrix

$$
\frac{1}{2}\left(A J+J^{T} A\right)
$$

( $\mathrm{J}$ is the Jacobian matrix of $\frac{\partial f}{\partial x}$ and $J^{T}$ its transpose) satisfy

$$
\lambda_{k} \leq-\delta<0, k=1,2, \cdots, n
$$

For all $(\mathrm{x}, \mathrm{t}) \in R^{n+1}$, where $\delta$ is a constant;

$$
\int_{t}^{t+r}\left\|f\left(\tau, 0, u^{*}\right)\right\| \rightarrow 0 \text { as } t \rightarrow \infty
$$

then the system (10) is asymptotically stable about the origin; and for each $x_{0} \in R^{n}$ the solution $\mathrm{x}(\mathrm{t})$ of (10) with $\mathrm{u}(\mathrm{t})=u^{*}(t)$ and $x\left(t_{0}\right)=x_{0}$ tends to $x_{1}=0$ as $t \rightarrow \infty$. If in addition to the above the linearized system:
$\dot{x}(t)=L(t) x(t)+q(t), q(t) \in Q(t) \subseteq R^{n}$

where $L(t)=f_{x}(t, 0,0)$ is an $\mathrm{n} \times \mathrm{n}$ matrix which is continuous in $\mathrm{t}, Q(t)=k(\wedge(t))$, where $k(\wedge)$ is the unbounded closed convex cone of $\Lambda$ with $\Lambda=f(t, 0, \Omega)$; is such that $0 \in \Omega, f(t, 0,0)=0$ and the system (27) is proper; i.e. the origin is an interior point of the reachable set $R\left(t, t_{0}\right)$, which is defined to be:

$$
R\left(t, t_{0}\right)=\left\{\int_{t_{0}}^{t} X^{-1}\left(s, t_{0}\right) q(s) d s: q(s) \in Q(s), q \text { summable }\right\}
$$

where $X\left(t, t_{0}\right)$ is the transition matrix of (27), then (10) is globally finite time null controllable, i.e. (10) is globally asymptotically stable and can be controlled to the origin with the control $u^{*}(t)$ in finite time from any initial position.

While linear approximation technique was used in [3] and [4] to determine the controllability of (10), we examine a different technique, perturbation method, used by Dauer [5] in determining the controllability of (10) with control, $\mathrm{u}(\mathrm{t})$, unrestrained. Dauer considered the system:

$$
\dot{x}(t)=A(t) x+B(t) u+f(t, x, u)
$$

where the continuous function $\mathrm{f}$ satisfies the condition:

$$
\lim _{|(x, u)| \rightarrow \infty} \frac{|f(t, x, u)|}{|(x, u)|}=0
$$

uniformly for $t \in\left[t_{1} t_{o}\right] \equiv I$. He showed that if the system:

$$
\dot{x}(t)=A(t) x+B(t) u
$$




$$
x\left(t_{0}\right)=x_{0}
$$

is completely controllable then the system (29) is completely controllable. Complete controllability of the system (31) implies the ability to steer any point of $E^{n}$ to another point of $E^{n}$ using unrestrained controls. This is equivalent to the positive definiteness of the Gramian matrix:

$$
W=\int_{I} X^{-1}(s) B(s) B^{*}(s) X^{-1 *}(s) d s
$$

where $\mathrm{X}(\mathrm{t})$ is the fundamental matrix solution of the uncontrolled system $(31)$ and $B^{*}(s)$ denotes the transpose of $\mathrm{B}(\mathrm{s})$. Dauer [6] extended this result to controllability of the perturbed nonlinear system:

$$
\dot{x}=g(t, x)+B(t, x) u(t)+f(t, x, u), \quad x\left(t_{0}\right)=x_{0}
$$

where $\mathrm{g}$ is twice differentiable in $\mathrm{x}$ and once in $\mathrm{t}, \mathrm{B}$ is once continuously differentiable in $\mathrm{x}$ and bounded in $I \times E^{n}$,

$\left|\frac{\partial g}{\partial x}\right|$ is bounded, and if $\mathrm{f}$ satisfies the conditions in (29), then (34) is completely controllable, if and only if:

$$
\dot{x}=g(t, x)+B(t, x) u(t)
$$

satisfies a strong controllability condition, i.e. if there exists a number $\lambda>0$ such that for any pair of continuous functions $\mathrm{x}(\mathrm{t}), \mathrm{u}(\mathrm{t})$ and for all $w \in E^{n}$ we have:

where:

$$
w^{*} s(x, u) w \geq \lambda|w|^{2}
$$

$$
\begin{aligned}
& s(x, u)=\int_{t_{0}}^{t_{1}} \psi(\tau) \psi^{*}(\tau) d \tau \\
& \psi(t)=z\left(t_{1}, t, x(t)\right) B(t, x(t))
\end{aligned}
$$

and where the Jacobi matrix function:

$$
z(t, s, x)=\frac{\partial y(t, s, x)}{\partial x}
$$

which is bounded on $I \times I \times E^{n}$ is the fundamental matrix solution of:

$$
\frac{\partial z}{\partial t}=\left(\frac{\partial g(t, y(t, s, x))}{\partial y(t, s, x)}\right) z .
$$

$\mathrm{z}(\mathrm{t}, \mathrm{t}, \mathrm{x})$ is the identity matrix and $y\left(t, s, x_{0}\right)$ is the unique solution of the nonlinear system:

$$
\begin{aligned}
& \dot{y}(t)=g(t, y) \\
& y\left(t_{0}\right)=x_{0}
\end{aligned}
$$

The symmetric and non-negative matrix $\mathrm{s}(\mathrm{x}, \mathrm{u})$ has a bounded inverse,

$$
\left|s^{-1}(x, u)\right| \leq \frac{1}{\lambda}
$$

Independently of $\mathrm{x}$ and $\mathrm{u}$.

We note that the controllability of (35) with restrained controls demands, in addition to the restraints on $\mathrm{g}$, local Lipchitz conditions on g, boundedness condition on B and f, stability results on (35). Similarly, the controllability of (29) with restrained controls requires the same bounds on B and $f$ as in (34) together with the stability conditions on the free system:

$$
\dot{x}=g(t, x)+f(t, x, 0) \text {. }
$$

The problem of constrained controllability thus reviewed entails the use of admissible controls which are constrained to lie in a unit cube. There is need to reformulate the controlled, unconstrained problem because in actual application there are hard limits on the structure which is needed to drive the system which is to be controlled.

We can generalize further the constrained controllability theory to accommodate additional uncertainty or disturbances. Into our new set we incorporate a restraining set $\mathrm{Q}$ within which the disturbance must lie. The results will then reduce to the earlier ones by simply taking the admissible parameter uncertainty $Q$ to be the singleton zero. The linear systems considered are given by:

$$
\dot{x}(t)=A(t) x(t)-p(t)+q(t)
$$

where for example $-\mathrm{p}(\mathrm{t})=\mathrm{B}(\mathrm{t}) \mathrm{u}(\mathrm{t})$ and $\mathrm{q}(\mathrm{t}) \in Q$. The system (45) can be considered as a pursuit game where $\mathrm{p}$ is the pursuer strategy and q the quarry strategy.

Isaacs [6] studied the pursuit game of two players moving on a straight line, the pursuer having a bound on his acceleration, the quarry a bound on his speed. The game ends when the pursuer attains a previously given distance from the quarry. The equations of motion are:

$$
\ddot{x}=u, \dot{y}=v
$$

$\mathrm{X}(\mathrm{t})$ two player system:

and in the matrix form:

$$
\dot{x_{1}}=x_{2}, \quad \dot{x}_{2}=u, \quad \dot{x_{3}}=v
$$

where:

$$
\dot{x}=A x+B u+C v
$$




$$
A=\left[\begin{array}{lll}
0 & 1 & 0 \\
0 & 0 & 0 \\
0 & 0 & 0
\end{array}\right], \quad B=\left[\begin{array}{l}
0 \\
1 \\
0
\end{array}\right], \quad C=\left[\begin{array}{l}
0 \\
0 \\
1
\end{array}\right]
$$

and:

$$
x=\left[\begin{array}{l}
x_{1} \\
x_{2} \\
x_{3}
\end{array}\right]
$$

or if we define $\mathrm{p}$ and $\mathrm{q}$ as in (45) we have

$$
\dot{x}=A x-p+q \text {. }
$$

The termination condition $|x-y|<\varepsilon$ translates to:

$$
\left(\begin{array}{l}
x_{1} \\
x_{2} \\
x_{3}
\end{array}\right) \in[-\varepsilon, \varepsilon] \text {. }
$$

The game is to drive the system to a target in $E^{n}$ using controls $\mathrm{p}(\mathrm{t})$ against all actions of the disturbance $\mathrm{q}(\mathrm{t})$.

Hajek [7] considered the linear differential game:

$$
\begin{aligned}
& \dot{x}(t)=A(t) x(t)-p(t)+q(t) \\
& x(t) \in C(t), p(t) \in P(t), q(t) \in Q(t) \\
& x(\text { end })=0
\end{aligned}
$$

wheret $\rightarrow A(t)$ is locally $L_{1}, t \rightarrow Q(t)$ is continuous and non-decreasing with $0 \in Q(t)$, and $\mathrm{P}(\mathrm{t})$ closed for all $t \geq 0$. He demonstrated that the game (54) is equivalent to the associated control system:

$$
\begin{aligned}
& \dot{x}(t)=A(t) x(t)-u(t) \\
& x(t) \in C(t), u(t) \in U(t) \\
& x(\text { end })=0
\end{aligned}
$$

where $\mathrm{A}, \mathrm{C}$ are as in (54) and the real state space $R^{n}, n \geq 0$, and the control constraint set is:

$$
U(t)=P(t)_{-}^{*} Q(t)
$$

${ }^{*}$ denotes the Pontriagin difference, $x \in V_{-}^{*} W$ if and only if $x+W \subseteq V$. By taking A and $\mathrm{Q}$ constant he extended this result to capture in target set $\mathrm{T}$ :

$$
T=\{x: M x=0\}
$$

$\mathrm{M}$ is a real mxn matrix.

Chukwu [8] considered the game (54) and extended Hajek's result to no-constant system matrix, $t \rightarrow A(t)$, locally $L_{1}, t \rightarrow Q(t), t \rightarrow P(t), t \rightarrow G(t)$ are non-empty set valued functions. He showed that the system steers into the target, $\mathrm{G}(\mathrm{t})$, against all actions of the disturbance provided the associated control system

The topics studied here are the non-linear differential equation analogues of these fundamental results of Hajek [7] and Chukwu [8], the first and second reciprocity theorems in [7] and G-controllability in [8]. The thrust of our result is that the non-linear pursuit problem (nonlinear control systems under square integrable but unpredictable perturbations) is reduced to one in control theory.

Let $E^{n}$ be the n-dimensional Euclidean space. For $t_{0}, t_{1} \in E$ with $t_{1} \geq t_{0}$, let the symbol $L_{2}\left(\left[t_{0}, t_{1}\right], E^{n}\right)$ denote the space of square integrable functions from $\left[t_{0}, t_{1}\right]$ into $E^{n}$. We examine the nonlinear differential system:

$$
\dot{x}(t)=f(t, x), x\left(t_{0}\right)=x^{0} \in E^{n}, t_{0} \in E\left(\cdot=\frac{d}{d t}\right)
$$

and the game described by:

$$
\dot{y}(t)=f(t, y)-p(t)+q(t), y\left(t_{0}\right)=x^{0}
$$

wherep $\in L_{2}\left(\left[t_{0}, t_{1}\right], P\right), P \subseteq E^{n}, q \in L_{2}\left(\left[t_{0}, t_{1}\right], Q\right), Q \subseteq E^{n}$ and where $f: E \times E^{n} \times E^{n} \rightarrow E^{n}$ is continuous. We shall assume as basic that the Frechet derivative $f_{x^{0}}\left(t, x^{0}\right)$ of $f\left(t, x^{0}\right)$ exists and is continuous in $\left(t, x^{0}\right)$, also $\mathrm{f}(\mathrm{t}, \mathrm{x})$ is Lipschitzian in each compact set of $E \times E^{n}$.We call $\mathrm{p}$ and q pursuer and quarry controls respectively if they are square integrable functions and satisfy $p(t) \in P, q(t) \in Q$ for all $t \in\left[t_{0}, t_{1}\right]$.

Lord and Mitchell [9] investigated the differential system:

$$
\dot{y}(t)=f(t, y)+F(t, y), \quad y\left(t_{0}\right)=x^{0} \in E^{n}
$$

where $f, F: E^{+} \times E^{n} \rightarrow E^{n}$ are continuous in (t, y), $E^{+}=(0, \infty)$. They demonstrated that if $x\left(t, t_{0}, x^{0}\right)$ is a unique solution of (61) such that $\frac{\partial x\left(t, t_{0}, x^{0}\right)}{\partial x^{0}}$ exists, is continuous and non-singular, then any solution $y\left(t, t_{0}, x^{0}\right)$ of (63) satisfies:

$$
y\left(t, t_{0}, x^{0}\right)=x\left(t, t_{0}, x^{0}\right)+\int_{t_{0}}^{t} \Phi\left(t, t_{0}, \phi(s)\right) \Phi^{-1}\left(s, t_{0}, \phi(s)\right) F(s, y(s)) d s
$$

where $\phi(t)$ is determined by the relation:

$$
\dot{\phi}(t)=\Phi^{-1}\left(t, t_{0}, \phi(t)\right) F\left(t, x\left(t, t_{0}, x^{0}\right)\right), \quad \phi\left(t_{0}\right)=x^{0}
$$


where $x\left(t, t_{0}, x^{0}\right)$ is the unique solution of (61). Furthermore, if $f_{x}(t, x)$, the derivative of $\mathrm{f}(\mathrm{t}, \mathrm{x})$ with respect to the solution $x\left(t, t_{0}, x^{0}\right)$ of $(61)$, exists and is continuous for $(\mathrm{t}, \mathrm{x})$ in $E^{+} \times E^{n}$ then the integral system (64) is equivalent to:

$$
y\left(t, t_{0}, x^{0}\right)=x\left(t, t_{0}, x^{0}\right)+\int_{t_{0}}^{t} \Phi(t, s, y(s)) F(s, y(s)) d s
$$

where $\frac{\partial x\left(t, t_{0} x^{0}\right)}{\partial x^{0}}=\Phi\left(t, t_{0}, x^{0}\right)$ is the fundamental matrix solution of the variational equation:

$$
\begin{aligned}
& \dot{z}(t)=f_{x}\left(t, x\left(t, t_{0}, x^{0}\right)\right) z(t) \\
& \Phi\left(t_{0}, t_{0}, x^{0}\right)=I(\text { identity matrix ) }
\end{aligned}
$$

that is:

$$
\Phi(t, s, y(s))=\Phi\left(t, t_{0}, \phi(s)\right) \Phi^{-1}\left(s, t_{0}, \phi(s)\right) .
$$
arises.

In view of the conditions imposed on $\mathrm{f}$ in (61) we shall make use of the form (66) whenever the need

\section{Definition 2.1}

There is complete capture everywhere at time $t_{1}$, for game (61) if for each $x^{0}, y^{l} \in E^{n}$ and for any quarry control $q \in L_{2}\left(\left[t_{0}, t_{1}\right], Q\right)$ there exists pursuer strategy $\sigma: Q \times\left[t_{0}, t_{1}\right] \rightarrow P$ which is $L_{2}\left(\left[t_{0}, t_{1}\right], P\right)$ satisfying:

i) $\quad p(t)=\sigma(q(t), t) \in P(t), q(t) \in Q(t)$

ii) The pair of controls p, q so obtained are such that the solution of the game (61) satisfies:

$y\left(t_{1}, t_{0}, x^{0}, p, q\right)=y^{1}$ and $y\left(t_{0}, t_{0}, x^{0}, p, q\right)=x^{0}$ with $y^{I}=0$.

There is capture in 0 at time $t_{l}$ for game (61) if the assumptions in the preceding definition are met

Capture in $\mathrm{G}$ at time $t_{1}$ is defined as follows: for each $x^{0} \in E^{n}$ and any quarry control $\mathrm{q}$, there exists a pursuer control $p \in L_{2}\left(\left[t_{0}, t_{1}\right], P\right)$ such that $y\left(t_{1}, t_{0}, x^{0}, p, q\right) \in G \subseteq E^{n}$ and $y\left(t_{0}, t_{0}, x^{0}, p, q\right)=x^{0}$.

Associated with our gameis a nonlinear control system described by the equation:

$$
\dot{y}(t)=f(t, y)-u(t), u(t) \in U, t \geq t_{0} \text {. }
$$

Here $\mathrm{f}$ is as in (61) but $u \in L_{2}\left(\left[t_{0}, t_{1}\right], U\right)$ where the control set is:

$$
U=P_{-}^{*} Q=\left\{y \in E^{n}: y+Q \subseteq P\right\} \text { (the Pontriagin difference of sets). }
$$

Definition 2.2

The system (71) is controllable if for each $y^{l} \in E^{n}$ there exists time $t_{1} \geq t_{0}$ and control $u \in$ $L_{2}\left(\left[t_{0}, t_{1}\right], U\right)$ such that the solution $y\left(t_{1}, t_{0}, x^{0}, u\right)$ of $(71)$ satisfies $y\left(t_{1}, t_{0}, x^{0}, u\right)=y^{l}$ and $y\left(t_{0}, t_{0}, x^{0}, u\right)=x^{0}$.

System (71) is null-controllable if the assumptions of the preceding definition are met with $y^{l}=0$.

\section{Main Results}

Let $\Phi\left(t, t_{0}, x^{0}\right)$ be the fundamental solution of the variational system:

$$
\dot{z}(t)=f_{x}\left(t, x\left(t, t_{0}, x^{0}\right)\right) z(t)
$$

with the standard assumptions of (61).

Proposition 3.1

Assume $0 \in Q(t)$ and $\mathrm{P}(\mathrm{t})$ closed for all $t \geq t_{0}$. There is complete capture everywhere at time $t_{1}$ for (61) if, and only if, the associated nonlinear control system:

$$
\begin{aligned}
& \dot{y}(t)=f(t, y)-u(t), y\left(t_{0}\right)=x^{0} \in E^{n} \\
& u(t) \in U(t) \equiv\left(P+\operatorname{ker} \Phi\left(t_{1}, t, y(t)\right)\right)_{-}^{*} Q
\end{aligned}
$$

Is controllable at time $t_{1}$. Furthermore, $\sigma(q, t)=u(t)+q$ modulo $\operatorname{ker} \Phi\left(t_{1}, t, y(t)\right)$, for all $q \in Q, t \in$ $\left[t_{0}, t_{1}\right]$,can be used to determine a suitable control strategy from an admissible control $u \in L_{2}\left(\left[t_{0}, t_{1}\right], U\right)$ for (74) and vice versa.

This is the nonlinear analogue to Hajek's [7] first duality theorem. We shall omit the proof of Proposition 3.1 since it is analogous to the more general situation in Proposition 3.2.

Proposition 3.2 
For the game (61), target set $G \subseteq E^{n}$, assume $0 \in Q$ and $\mathrm{P}$ closed. There is capture in $\mathrm{G}$ at time $t_{1}$ for (61) if, and only if, the associated nonlinear control system:

$$
\begin{aligned}
& \dot{y}(t)=f(t, y)-u(t), u(t) \in U, t \geq t_{0} \\
& y\left(t_{0}\right)=x^{0} \in E^{n} \\
& u(t) \in U(t) \equiv\left(P+\operatorname{ker} M \Phi\left(t_{1}, t, y(t)\right)\right)
\end{aligned}
$$

Is G-controllable at time $t_{1}$. Moreover, $\sigma(q, s)=u(s)+q$ modulo ker $M \Phi\left(t_{1}, s, y(s)\right)$ may be used to determine an appropriate control strategy from $u \in L_{2}\left(\left[t_{0}, t_{1}\right], U\right)$ in (76) and vice versa.

Proof

Assume that there is a capture in $\mathrm{G}$ of the game (61) at time $t_{1}$, then for any $q \in L_{2}\left(\left[t_{0}, t_{1}\right], Q\right)$, any $x^{0} \in E^{n}$,some p, $p(t)=\sigma(q(t), t) \in L_{2}\left(\left[t_{0}, t_{1}\right], P\right)$ the solution $y\left(t_{1}, t_{0}, x^{0}, p, q\right)=y\left(t_{1}\right)$ satisfies:

$$
M y\left(t_{1}\right)=M\left(x\left(t_{1}\right)-\int_{t_{0}}^{t_{1}} \Phi\left(t_{1}, s, y(s)\right)(\sigma(q(s), s)-q(s)) d s\right)=M b
$$

For quarry control $0(0 \in Q)$

$$
M\left(x\left(t_{1}\right)-\int_{t_{0}}^{t_{1}} \Phi\left(t_{1}, s, y(s)\right) u(s) d s=M b\right.
$$

where $u(s)=\sigma(0, s)$. By using piecewise constant quarry control q, q in $\left[t_{0}, t\right)$ and 0 in $\left[t_{,} t_{1}\right]$ and subtracting the result from $(80)$ yields:

$$
M \int_{t_{0}}^{t_{1}} \Phi\left(t_{1}, s, y(s)\right)(u(s)+q-\sigma(q, s)) d s=0
$$

for all $t \in\left[t_{0}, t_{1}\right]$, and hence $M \Phi\left(t_{1}, s, y(s)\right)(u(s)+q-\sigma(q, s))=0$ for almost all s from which we obtain:

$$
u(s) \in\left(P+\operatorname{ker} M \Phi\left(t_{1}, s, y(s)\right)\right)_{-}^{*} Q \text { a.e. }
$$

Hence $u \in L_{2}\left(\left[t_{0}, t_{1}\right], U\right)$ is an admissible control for (76), and (80) yields $M y\left(t_{1}\right)=M b$, proving that (76) is

G-controllable at $t_{1}$.

Conversely, assume that (76) is G-controllable at time $t_{1}$ and let $x^{0} \in E^{n}, b \in E^{n}$ be given. Let u be the appropriate control in $L_{2}\left(\left[t_{0}, t_{1}\right], U\right)$ such that $y\left(t_{0}\right)=x^{0}, M y\left(t_{1}\right)=M b$, then:

where $u(s) \in U(s)$ yields:

$$
M b=M\left(x\left(t_{1}\right)-\int_{t_{0}}^{t_{l}} \Phi\left(t_{1}, s, y(s)\right) u(s) d s\right)
$$

$u(s)+q(s) \in\left(P+\operatorname{ker} \mathrm{M} \Phi\left(t_{1}, s, y(s)\right)\right.$.

We now apply Filipov's lemma (see form in Hajek [10]) to construct a pursuer control: there exist measurable mappings $\sigma: Q \times\left[t_{0}, t_{l}\right] \rightarrow P, v: Q \times\left[t_{0}, t_{l}\right] \rightarrow \operatorname{ker} M \Phi\left(t_{l}, ;, y(\cdot)\right)$ such that $u(s)+q(s)=\sigma(q, s)+v(q, s)$. Because $\sigma$ takes values in a closed set $\mathrm{P}, \sigma \in L_{2}\left(\left[t_{0}, t_{1}\right], P\right)$. We now show that for any quarry control q, $\sigma$ is indeed appropriate; for any $q \in L_{2}\left(\left[t_{0}, t_{1}\right], Q, \sigma-q=u-v\right.$, so that the solution at $t_{1}$ of (61) with this pair of $\sigma$ and q with initial data $x^{0}$ is:

$$
M y\left(t_{1}\right)=M\left(x\left(t_{1}\right)-\int_{t_{0}}^{t_{l}} \Phi\left(t_{1}, s, y(s)\right)(\sigma(q, s)-q(s)) d s\right)
$$

Corollary 3.3

Under the assumptions of Proposition 3.2, there is capture in 0 at time $t_{1}$ for game (61) if, and only if, the control system (76) is null-controllable.

Proof

Take $\mathrm{M}=\mathrm{I}$ (the identity matrix) and $b \in E^{n}$ with $b \equiv 0$. This completes the proof.

Example 3.4

Consider the equation of the forced pendulum with motion constrained to the plane and mass $\mathrm{m}$ attached to the pivot by means of a rigid massless member:

$$
I \ddot{\theta}+\alpha \dot{\theta}+m g l \sin \theta=H(t)
$$

where $I=m l^{2}$ and $\mathrm{H}(\mathrm{t})$ is the external controlling torque, $\alpha \geq 0$ is the damping coefficient. A more general nonlinear version of (87) can be considered to be:

$$
\ddot{\theta}+f(\theta, \dot{\theta}) \dot{\theta}+g(\theta)=\beta(t)-\rho(t)
$$

where $\beta(t)$ is the external controlling torque and $\rho(t)$ is the regulating torque and where $\mathrm{f}$ and $\mathrm{g}$ satisfy the conditionsin (18) and (19). The termination condition is $(\theta=0=\dot{\theta})$ subject to the constraints $|\beta(t)| \leq 1$, $|\rho(t)| \leq \rho_{0}$ and $t \geq 0$.We express (87) with $x=\theta, \dot{x}=y$ in the phase plane form to obtain:

$$
\begin{aligned}
& \dot{x}=y \\
& \dot{y}=-f(x, y) y-g(x)+\beta(t)-\rho(t)
\end{aligned}
$$


Or in the matrix form as:

$$
\dot{X}=\left(\begin{array}{c}
y \\
-f(x, y) y-g(x)
\end{array}\right)+\left(\begin{array}{l}
0 \\
1
\end{array}\right) \beta(t)-\left(\begin{array}{l}
0 \\
1
\end{array}\right) \rho(t)
$$

where $X=\left(\begin{array}{l}x \\ y\end{array}\right)$ and

$$
F(x, y)=\left(\begin{array}{c}
y \\
-f(x, y) y-g(x)
\end{array}\right)
$$

We isolate the nonlinear part of (90) as:

$$
\dot{X}=\left(\begin{array}{c}
y \\
-f(x, y) y-g(x)
\end{array}\right)
$$

Let $\mathrm{x}$ be the unique solution of $(92)$ then

$$
F_{x}(x, y)=\left(\begin{array}{cc}
0 & 1 \\
-\dot{g}(x)-f_{x}(x, y) y & -f(x, y)-f_{y}(x, y) y
\end{array}\right)
$$

We solve the variational equation:

$$
\dot{z}(t)=\left(\begin{array}{cc}
0 & 1 \\
-g(x)-f_{x}(x, y) y & -f(x, y)-f_{y}(x, y) y
\end{array}\right) z(t)
$$

to obtain $\Phi(t)$, the fundamental matrix solution of (94). We now apply Proposition 3.1 to the game to a problem in control. We assume $\rho_{0}<1$; then there is a winning strategy $\beta=\rho+u$ where uis the control which steers to the origin within the control system:

$$
\dot{y}+f(x, y) y+g(x)=u
$$

The player constraint sets in $E^{2}$ are:

$$
P=\left\{\left(\begin{array}{l}
0 \\
\beta
\end{array}\right):|\beta| \leq 1\right\}, \quad Q=\left\{\left(\begin{array}{l}
0 \\
\rho
\end{array}\right):|\rho| \leq \rho_{0}\right\}
$$

So that since $\Phi(t)$ is a fundamental matrix solution it is nonsingular and has zero kernel, we have:

$$
(P+\operatorname{ker} \Phi(t))_{-}^{*} Q=P_{-}^{*} Q=U=\left\{\left(\begin{array}{l}
0 \\
u
\end{array}\right):|u| \leq 1-\rho_{0}\right\}
$$

We see that the nonsingular game can be steered to the target if the conditions in (18) and (19) hold.

\section{Conclusion}

We see that in establishing the nonlinear differential equation analogues of the first and second reciprocity theorems of Hajek and G-controllability result of Chukwu, we have demonstrated that the nonlinear pursuit

\section{References}

[1]. Hermes, H. and Lasalle, J. P., Functional Analysis and Time Optimal Control (Academic Press, 1969)

[2]. Lasalle, J. P., The Time Optimal Control Problems, Annals of Mathematics, Vol. 45, 120-124, 1966

[3]. Lee, E. B. and Markus, L., Foundations of Optimal Control Theory (John Wiley and Sons, 18-29, 1967)

[4]. Chukwu, E. N., Finite time controllability of nonlinear control processes, Siam J. Control, Vol. 13, No 4, 807-816, 1975

[5]. Hajek, O., Duality for differential games and optimal control,Maths systems Theory, Vol. 8,No 1,1-7,1974

[6]. Chukwu, E. N., Controllability to a target set of linear disturbed systems, IEEE, No 4,Vol. AC-22,625-626,1977

[7]. Lord, M. E. and Mitchell, A. R., A new approach to the method of nonlinear variation of parameters, University of Texas, No. 18, 1-12, 1975 